\section{Schadenersatz für liquidationsberechtigten Arzt? Ausübung des Liquidationsrechts war zeitweise nicht möglich}

Das Landesarbeitsgericht (LAG) Düsseldorf wies die Klage eines liquidationsberechtigten Arztes auf Schadenersatzansprüche wegen Unmöglichkeit der Durchführung des Liquidationsrechts, weil ihm die erforderlichen Mitarbeiter nicht zur Verfügung gestellt wurden, ab (Urteil vom 20.04.2015 Az.: 9 S a 151/15).

\section{Der Fall}

Die Beklagte betreibt eine medizinische Einrichtung, in der Patienten sowohl stationär als auch ambulant (in einem MVZ) versorgt werden. Bei dem Kläger handelt es sich um einen Arzt, der seit 15.06.2011 in der Einrichtung beschäftigt ist. Hierzu existieren 2 Arbeitsverträge mit der Beklagten. In einem Vertrag ist der Kläger als Facharzt für Nuklearmedizin mit einer Arbeitszeit von 32 Stunden pro Woche im MVZ tätig. In einem zweiten Vertrag ist er im stationären Bereich für 8 Stunden pro Woche als Oberarzt in der Klinik für Strahlenheilkunde und Nuklearmedizin angestellt. Zu beiden Verträgen existieren Nebenabreden. Danach erhält der Kläger neben seinem Grundgehalt 45\% der Privatambulanzerlöse sowie bei Wahlleistungspatienten das Honorar abzüglich der dem Krankenhaus entstehenden Kosten.

Zur Erbringung von nuklearmedizinischen Leistungen wurden dem Kläger 2 Mitarbeiterinnen zugeordnet. Eine der Mitarbeiterinnen war zu Beginn des Jahres 2014 durchgehend arbeitsunfähig erkrankt, die andere befand sich im Juli 2014 auf Fortbildung bzw. im Urlaub. Der Kläger forderte die Beklagte mehrfach erfolglos auf, für diesen Zeitraum Ersatzpersonal zu stellen. In der Folge behandelte der Kläger im Bereich der Nuklearmedizin in dem vorgenannten Zeitraum keine Patienten.

\section{Vorübergehend keine Mitarbeiter \\ - Arzt klagt auf Schadenersatz}

Hierfür verlangte der Kläger beim Arbeitsgericht Schadenersatz für entgangene Vergütung aus Privatliquidation in dem entsprechenden Zeitraum. Er begründete seine Klage damit, dass die Beklagte ihm für diesen Zeitraum das erforderliche nichtärztliche Personal nicht zur Verfü- gung gestellt habe, obwohl bereits seit Anfang 2014 der krankheitsbedingte Ausfall einer der ihm zuarbeitenden Kräfte bekannt gewesen sei. Ohne das nichtärztliche Personal sei es ihm aber nicht möglich, den Betrieb im Bereich Nuklearmedizin aufrechtzuerhalten. In erster Instanz gab ihm das Arbeitsgericht Duisburg im Wesentlichen recht. Das Gericht bestätigte dem Kläger zunächst einen Anspruch auf Schadenersatz für Liquidationen/Beteiligungen, die ihm dadurch entgangen seien, dass er im Zeitraum vom 04.07.2014 bis 25.07.2014 stationäre Patienten der Beklagten bzw. ambulante Patienten im MVZ aufgrund des vollständigen Ausfalls des nichtärztlichen Personals nicht habe behandeln können. Die Beklagte habe insoweit ihre vertragliche Nebenpflicht verletzt, weil dem Kläger vertraglich das Recht zur Privatliquidation eingeräumt worden sei. Damit habe sie die Verpflichtung übernommen, ihm die personellen und sächlichen Mittel zur Verfügung zu stellen, die dieser zur Behandlung der Patienten benötige. Das erstinstanzliche Gericht vertrat daher die Auffassung, als Arzt sei der Kläger auf Mitarbeiter, die Hilfstätigkeiten bzw. unterstützende Arbeiten leisteten, angewiesen. Die Pflichtverletzung habe die Beklagte auch durch ihr Organisationsverschulden zu vertreten. Gegen dieses Urteil ging die medizinische Einrichtung in Berufung.

\section{LAG hebt Urteil auf und weist Klage ab}

Die Richter am LAG hoben das erstinstanzliche Urteil auf und wiesen die Klage ab. Dem Kläger stünden gegenüber der Beklagten in dem betreffenden Zeitraum keine weitergehenden Zahlungsansprüche zu. Auch wenn eine generelle Pflicht der
Beklagten bestehen sollte, dem Kläger die Mittel zur Verfügung zu stellen, die er zur Behandlung seiner Patienten benötigt, werde allein durch die Erfüllung eines berechtigten Urlaubswunsches einer Mitarbeiterin kein Schadensersatzanspruch ausgelöst. Selbst dann, wenn dieser dazu führt, dass aufgrund des krankheitsbedingten längerfristigen Ausfalls eines weiteren Mitarbeiters vorübergehend keine ärztliche Tätigkeit durchgeführt werden könne. Die Beklagte habe ihre arbeitsvertraglichen Nebenabreden nicht verletzt. Als mögliche Anspruchsgrundlage kam laut LAG bei den hier vorliegenden Vereinbarungen ausschließlich Schadenersatz wegen Pflichtverletzung ( $§ 280$ Abs. 1 BGB) in Betracht, dessen Voraussetzungen allerdings nicht vorlägen.

\section{Keine Pflichtverletzung}

Nach der Regelung kann der Gläubiger (in diesem Fall der Kläger) Ersatz des Schadens verlangen, wenn der Schuldner (in diesem Fall die medizinische Einrichtung) eine Pflicht aus dem Schuldverhältnis (hier die Nebenabreden zur Privatliquidation) verletzt. Dies gilt allerdings dann nicht, wenn der Schuldner die Pflichtverletzung nicht zu vertreten hat. Selbst wenn unterstellt werde, dass der Kläger ohne seine beiden ihm zugeordneten Mitarbeiter tatsächlich keine unmittelbare Behandlung am Patienten durchführen konnte, scheide eine Haftung aus. Denn aus Sicht der Kammer fehle es schon an einer Pflichtverletzung, jedenfalls aber am Verschulden. Auch wenn der Kläger mindestens eine Mitarbeiterin zur Ausführung der unmittelbaren ärztlichen Aufgaben benötige, die dann aufgrund der Umsatzbeteiligung vergütungswirksam seien, liege keine Pflichtverletzung vor, wenn die Beklagte dem Kläger für den Zeitraum der vorübergehenden urlaubsbedingten Abwesenheit eines Mitarbeiters keine Ersatzkraft stellt. Denn der Arbeitgeber mache dem Arbeitnehmer nicht auf Dauer die Erzielung des weiteren Verdienstes unmöglich.

\section{Keine Pflicht für Ersatzpersonal wegen Urlaubs}

Auch wenn die Beklagte grundsätzlich die Pflicht habe, dem Kläger die Behandlung von bestimmten Patienten zur Erzielung einer weiteren Vergütung möglich zu machen, bestehe diese Pflicht von vornherein nur im Umfang der betrieblichen Organisation. Schadenersatzansprüche kämen von vornherein nicht in Betracht für Zeiten, in 
denen Mitarbeiter aufgrund zwingender arbeitsrechtlicher Regelungen nicht zur Erbringung der Arbeitsleistung zur Verfügung stünden. Eine Pflicht, während Urlaubszeiten eine Ersatzkraft zu stellen, nur damit dem Arbeitnehmer Tätigkeiten ermöglicht werden, die zusätzliche Einnahmen generieren, bestünde nicht. Denn die Erfüllung berechtigter Urlaubsansprüche könne nicht zu einem Schadenersatzanspruch anderer Mitarbeiter führen, selbst wenn die Erfüllung dieser Ansprüche bei den betroffenen anderen Mitarbeiter auch zu einer geringeren Vergütung führen möge, wenn er in diesem Zeitraum aufgrund der Abwesenheit eines anderen Mitarbeiters bestimmte vergütungsrelevante Arbeiten nicht ausführen könne.

\section{Ausnahme bei Personalunterdeckung}

Etwas anderes könne allenfalls gelten, wenn der Arbeitgeber den Betrieb so organisiert habe, dass durch den Urlaub stets eine Personalunterdeckung eintritt. So läge der Fall hier aber nicht. Da also der Arbeitgeber weder den Urlaub verweigern, noch eine Ersatzkraft einstellen musste, fehlte es bereits an der Pflichtverletzung.

\section{Kein Verschulden}

Auch ein Verschulden der Beklagten läge nicht vor. Die Beklagte habe den Betrieb grundsätzlich so organisiert, dass die Erbringung der vollständigen Arbeitsleistung möglich bleibe, wenn der Arbeitgeber berechtigte Freistellungsansprüche erfülle. Auch wenn der Urlaub mit einer Erkrankung des weiteren Mitarbeiters zusammenträfe, scheide ein Verschulden des Arbeitgebers aus. Denn der Arbeitgeber sei nicht verpflichtet, in einem derartigen Fall eine Ersatzkraft zu stellen. Allenfalls dann, wenn es ihm ohne Weiteres möglich wäre, eine Ersatzkraft zu finden und diese auch einzustellen, wäre es denkbar, auch in einem derartigen Fall ein Handeln des Arbeitgebers zu fordern. So habe der Fall hier aber nicht gelegen. Zum einen habe es sich um einen speziellen Bereich gehandelt, in dem Ersatzkräfte nicht ohne Weiteres zu rekrutieren gewesen sei und auch eine Personalreserve nicht vorbehalten werden müsse.

\section{Betriebsorganisation ist Organisa- tionshoheit des Arbeitgebers}

Denn es obliege der Organisationshoheit des Arbeitgebers, wie er seinen Betrieb organisiere. Er müsse nicht für jeden Be- reich eine Personalreserve vorhalten, solange er durch sein Organisationskonzept einen geregelten Ablauf ermöglicht. Dies aber sei der Fall, wenn sich im vom Kläger betreuten Bereich 2 Mitarbeiter vertreten. Dass nun eine der Kräfte krankheitsbedingt ausgeschieden sei, könne ein Verschulden auch dann nicht begründen, wenn eine Kraft länger arbeitsunfähig ausfalle und vorhersehbar sei, dass der anderen Hilfskraft im Laufe des Jahres der Jahresurlaub erteilt werden müsse. Denn solange der Arbeitgeber mit der Rückkehr des erkrankten Arbeitnehmers rechnen müsse, sei es ihm nicht zumutbar, insoweit unmittelbar eine Ersatzkraft einzustellen, die er dann mit der Rückkehr der erkrankten Mitarbeiterin wieder entlassen müsste. Hierzu könnte der Arbeitgeber nur dann verpflichtet sein, wenn er auf sicherer Prognosegrundlage weiß, dass der erkrankte Mitarbeiter nicht mehr an den Arbeitsplatz zurückkehren werde. So liege der Fall hier aber nicht.

\section{Kein Annahmeverzug}

Das LAG prüfte auch, ob das Liquidationsrecht des Arztes Gegenleistungscharakter für seine Arbeitstätigkeit habe - und daher u.U. die Beklagte sich im sog. Annahmeverzug befand ( $\$ 615$ BGB). Nach Auffassung des LAG muss diese Frage jeweils anhand des Einzelfalls und der zwischen den Parteien getroffenen Vereinbarungen geprüft werden.

Nach Auslegung der Vereinbarung über die Tätigkeit als stationärer Oberarzt kam das LAG zu dem Ergebnis, dass es in der Vereinbarung nicht darum ging, dem Liquidationsrecht des Klägers im Bereich der stationären Leistungen Gegenleistungscharakter zukommen zu lassen. In der Gesamtschau der Vertragslage werde deutlich, dass dem Kläger mit dem Liquidationsrecht nur noch einmal zusätzliche Einnahmen verschafft werden sollten. Denn er sei für eine angemessene Honorierung nicht auf diesen Liquidationsteil angewiesen gewesen, was bereits die Höhe der tatsächlichen aus diesem Liquidationsbestandteil erzielten Vergütung zeige (durchschnittlich 709,84 EUR monatlich). Dieser Betrag könne die Honorierung angesichts des üppigen fixen Vergütungsanteils aus dem zeitgleich abgeschlossenen Arbeitsvertrag über die Tätigkeit im MVZ nicht entscheidend beeinflussen. Es gehe erkennbar nicht um effektive Vergütung der Hauptleistung sondern ersichtlich darum, den „Status“ des Klägers im betrieblichen Gefüge durch das ihm eingeräumte Liquidationsrecht zu erhöhen.

Auch aus der Nebenabrede als angestellter Facharzt im MVZ stünde dem Kläger kein Anspruch aus $\S 615$ BGB zu. Denn es handele sich bei der Beteiligung an Erlösen der Privatpatienten im Rahmen der Nebenabrede um eine echte „Umsatzbeteiligung“, die neben der vereinbarten Vergütung fällig werde. Die Beklagte habe dem Kläger eine zusätzliche Vergütung zum Grundgehalt eingeräumt. Da der Umsatz als Berechnungsgrundlage für die zusätzliche Vergütung herangezogen werde, handele es sich letztlich um eine Mischform zwischen Tantiemen und Provision. Nach Auffassung des Gerichts handelt es sich zwar in diesem Fall um einen Teil des Entgelts für die vertraglich geschuldete Arbeitsleistung. Damit habe diese Vergütung Gegenleistungscharakter. Dennoch scheide ein Anspruch aus $\S 615$ BGB aus, weil im konkreten Zeitraum die Situation des Annahmeverzugs nicht gegeben war. Dies deshalb, weil der Kläger im relevanten Zeitraum durchaus Tätigkeiten aus seinen beiden Tätigkeitsspektren durchführen konnte, für die er aus seiner Sicht seine Mitarbeiter nicht benötigte. Dies habe der Kläger auch selbst dadurch zu erkennen gegeben, dass er in dieser Situation nicht „zu Hause geblieben“ sei.

\section{Fazit}

Das Urteil macht deutlich, dass keine pauschale Beurteilung derartiger Fälle erfolgen kann, sondern immer der Einzelfall und die jeweilige zugrunde liegende Vereinbarung sowie die betriebliche Organisationsstruktur geprüft werden müssen. Den medizinischen Einrichtungen wird insoweit ein verhältnismäßig großer Organisationsspielraum eingeräumt, der allerdings dort endet, wo z.B. regelmäßig eine Personalunterdeckung bewusst in Kauf genommen wird oder absehbar ist, dass es sich um eine dauerhafte Verhinderung der Ausübung des Liquidationsrechts handeln kann.

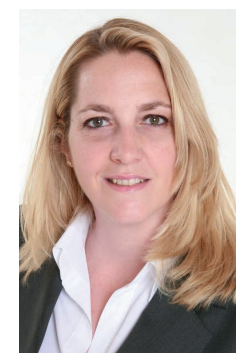

Korrespondenz Dr. iur. Isabel Häser Rechtsanwältin ETL Lüdemann Wildfeuer \& Partner Sonnenstr. 9 80331 München www.etl.de 